\title{
Online informational bariatric seminars: increasing access to bariatric surgery or widening a divide?
}

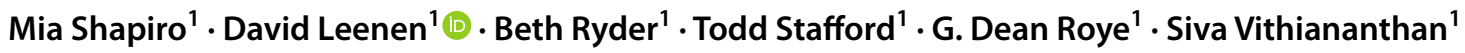

Received: 17 November 2020 / Accepted: 11 March 2021 / Published online: 29 March 2021

(c) The Author(s), under exclusive licence to Springer Science+Business Media, LLC, part of Springer Nature 2021

\begin{abstract}
Background Historically our institution has required prospective bariatric patients attend an in-person introductory seminar prior to their first clinic visit. Utilization of the internet has increased dramatically over the last 10 years and in 2016, we added an online seminar. With the emergence of COVID-19 and new rules regarding social distancing, we are currently relying exclusively on web-based orientation. Research suggests there are racial and socioeconomic disparities in the utilization of internet health resources in the general population. To our knowledge this has not been studied in regard to the bariatric population.

Methods A retrospective chart review was performed on patients who attended online (OS) and in-person seminars (IPS). Our primary endpoint was to compare the percentage of patients who underwent surgery. Secondary outcomes included differences in demographic and clinical characteristics between the patients who attended the OS versus the IPS.

Results Of the 1152 patients who attended a bariatric orientation, $71.2 \%$ scheduled an office visit. The IPS patients had a higher rate of office visits and shorter time between orientation and first visit. Of patients eligible for surgery, there was a higher percentage of Caucasians in the OS group while the IPS group had higher rates of Hispanic and African Americans. Patients from the OS group had higher rates of marriage and employment, and lower rates of medical co-morbidities. When controlling for age, sex, race/ethnicity, marital status, employment status and medical co-morbidities, there was no significant impact of type of orientation on progression to surgery.

Conclusion We found that there was no difference in progression to surgery between the two orientation groups. Importantly, however, we detected marked demographic and clinical differences between the two patient populations. There is an urgent need to address these disparities as we are now heavily relying on patient outreach and education via the internet.
\end{abstract}

Keywords Obesity $\cdot$ Bariatric surgery $\cdot$ Outcomes

Between 2017 and 2018, the age-adjusted prevalence of obesity $(\mathrm{BMI}>30)$ in adults in the United States was $42.8 \%$ and that of severe obesity (BMI $>40$ ) was $9.2 \%$ [1]. Patients with a BMI $>40$ qualify for elective bariatric surgical intervention but in 2018 only 228,000 patients underwent bariatric surgery [2]. Improving knowledge regarding the benefits of surgery and access to intervention is a health priority. At our institution, as in many others across the country, patients are required to attend an information session prior to their first office visit. Traditionally, these sessions have been offered as in-person group orientations. A Pew Research Center

Siva Vithiananthan svithi1@gmail.com

1 Miriam Hospital/Brown University, 195 Collyer St. Suite 302, Providence, RI 02904, USA report states that as of $2012,81 \%$ of US adults reported using the internet and $72 \%$ of those people had searched for health information [3]. In response to this trend, we created an alternative online orientation. With COVID-19 cases on the rise across the country, we have had to put our in-person seminars on hold and all orientation sessions have been converted to a web-based platform. The goal of our study was to determine if there were any differences in progression to surgery between the groups who chose the online bariatric session versus the in-person seminar. As digital literacy in the US has been shown to vary based on age, race/ethnicity and education levels, we additionally hoped to uncover any disparities in demographic and clinical characteristics between the two populations. 


\section{Materials and methods}

Since the start of our integrated bariatric surgery program in 2012, we have required that each patient attend an inperson seminar prior to their first clinician visit. Sessions are offered every 2 weeks and last approximately $2 \mathrm{~h}$. They are given by either one of the bariatric surgeons or the nurse manager. Information on the prevalence of obesity and associated health problems are discussed, followed by the medical and surgical options and an opportunity to ask questions. The presentations are offered in English with Spanish and Portuguese interpreters present to assist patients with poor English literacy. After the IPS, patients are able to register for a clinic visit.

In July 2016, an online alternative to the in-person orientation was created. It consists of the same informational slides as the in-person seminar but requires English literacy, as it is only offered in English. After patients review the slides, they are asked to complete a post-presentation quiz. Patients with a passing score of $\geq 70 \%$ are called to schedule an in-person clinic appointment. Patients with a lower score ( $<5 \%$ of patients) are asked to attend an inperson informational session first. Both the in-person and online seminar patients are given one year to schedule an appointment.

We obtained an IRB waiver for a retrospective chart review of all patients who attended either an in-person (IPS) or online seminar (OS) through our bariatric center from the start of our online bariatric seminar program in July 2016 until December 2017. Our primary endpoint was the percentage of patients from each orientation group who elected to undergo surgery. The cut off for date of surgery was January 31, 2019 when we initiated our chart review. Secondary outcomes included differences in demographic and clinical characteristics of those who chose the IPS versus the OS and attended an office visit. Patients were excluded if they had had previous bariatric surgical intervention, had attended both the in person and the online orientations or were otherwise deemed ineligible for surgery based on initial assessment by the surgeon. Values are presented as percentages, means \pm standard deviation or interquartile ranges and compared using t-tests, chi-square analysis. We used regression modeling to determine whether or not surgical intervention varied based on attendance of the OS vs. IPS, controlling for various demographic and clinical factors including age, body mass index (BMI), gender, ethnicity, language, marital status, employment status, previous abdominal surgery and medical and psychological comorbidities. Age and BMI were treated as continuous covariates in our model and all other were treated as categorical covariates. All analysis was performed using SAS.

\section{Results}

During the 18-month period, 1152 eligible patients attended a bariatric orientation. The IPS was more popular with 770 $(66.8 \%)$ patients attending the IPS versus $382(33.2 \%)$ patients attending the OS $(p<0.001)$. The median age of the patients attending the IPS was $43.9 \pm 12.3$, and of the patients attending the OS was $36.2 \pm 11.2(p<0.001)$. The IPS consisted of $77.6 \%$ females, while the OS was $85.4 \%$ female $(p=0.002)$. While the IPS attendance varied over time with no obvious pattern, the OS showed an increased trend in popularity over the 18 months (Fig. 1).

Of the 1152 attendees, $71.2 \%$ scheduled an office visit following their orientation. Attendees of the IPS had a higher rate of office visits when compared to those who elected the OS $(80.1 \%$ vs $53.1 \%, p<0.001)$ and the median time from orientation to first office appointment was shorter for the IPS when compared to the OS (34 days, inter-quartile range (IQR, 19-51) vs 48 days, (IQR 36-68; $p<0.001$ ).

Of the 820 patients who scheduled an office visit, 719 were eligible for surgery based on their initial assessment by the surgeon. Table 1 illustrates the demographics and clinical characteristics of patients who attended an office visit and were eligible for surgery, stratified by orientation type. Patients eligible for surgery who utilized the OS group were younger on average, with median age of 36.2 years \pm 11 vs 44.1 years $\pm 12 ; p<0.001)$ compared to the IPS group. The percentage of women attending the OS was higher than that attending the IPS $(87.2 \%$ vs. $79.3 \% ; p=0.02)$. In regards to ethnicity, there was a higher percentage of Caucasians in the OS group ( $82.2 \%$ vs. $57.9 \%$ ), while the IPS had higher rate of Hispanic and African American (10.3\% vs. $27.2 \%$ and $5.2 \%$ vs. $11.8 \%$, respectively; $p<0.001$ for all). In regards to language, $2.2 \%$ of the OS group reported Spanish as their primary language, while $12.3 \%$ of the IPS group reported their primary language as Spanish $(p<0.001)$. Patients from the OS had higher rates of marriage $(54.0 \%$ vs $42.9 \%$; $p=0.037)$ as well as higher rates of employment $(82.7 \%$ vs $71.1 \% ; p=0.003)$. In regards to clinical characteristics, patients who chose the OS had significantly lower rates of diabetes ( 18.8 vs $29.3 \% ; p=0.003$ ), hypertension ( $35.8 \mathrm{vs}$ $54.5 ; p<0.001)$ and obstructive sleep apnea (49.7 vs 61.7; $p<0.006)$. There were no statistically significant differences noted in BMI, rate of previous abdominal surgery or history of depression/anxiety.

Because the online orientation was only offered in English, we performed a secondary analysis to see if the socioeconomic and clinical disparities we revealed could be attributed to the presence of the Spanish speaking population in the IPS group. We repeated the above analysis with English speakers only (Supplemental Table S1). We found the same significant trends in everything except for gender. While 


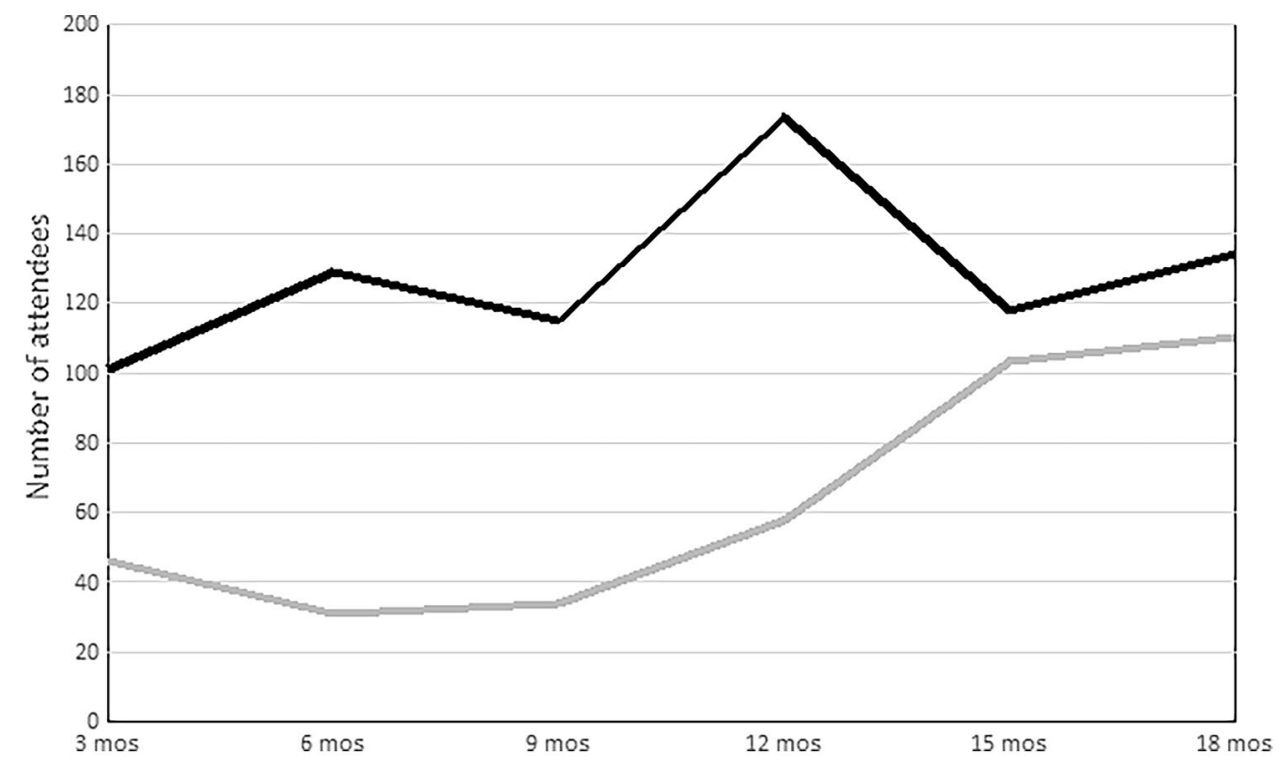

Fig. 1 Number of orientation attendees per 3 month period since inception of online orientation Online Seminar (OS) In Person Seminar (IPS)

there were more females attending the OS when compared to the IPS ( 86.7 vs $80.2 \% ; p=0.06$ ) the $p$ value did not quite reach significance.

Ultimately, 50.8\% (365/719) of the patients who attended an office visit after orientation underwent surgery. $52 \%$ of the patients who attended the OS and $50.2 \%$ of the patients who attended the IPS underwent surgery. When controlling for age, BMI, gender, ethnicity, language, marital status, employment status, previous surgery and medical and psychological comorbidities, there was no statistically significant impact of type of orientation on progression to surgical intervention (OR 1.227, 95\% CI 0.807-1.865; $p=0.339$ ).

\section{Discussion}

The goal of our study was to evaluate the progression to surgery between patients who attended either an in-person or an online informational seminar. We found that patients attending the online seminar were significantly less likely to schedule an office visit, however once the patients were seen in the office there was no difference in the percentage of patients who progressed to surgery. This is on par with other work which demonstrates that online bariatric orientations lead to similar or fewer office consultations $[4,5]$. It is possible that some of the people who attend the online informational seminar are less motivated to complete the process, as it is certainly easier to peruse an online orientation than it is to attend an in-person seminar.

More interestingly, the data from our bariatric cohort uncovered important socioeconomic and clinical disparities between the populations choosing the OS vs the IPS. Patients who chose the OS were younger and more likely to be female, Caucasian, married and employed. The patients who attended the OS were also on average healthier, with lower rates of comorbidities, including diabetes, hypertension and sleep apnea. A study by Eaton et al. compared groups attending an online bariatric session to an in-person bariatric session, and similarly found that the patients attending their online orientation were younger and had lower levels of comorbidities like diabetes and HTN [6].

There has been a general concern that health disparities have been magnified by the introduction of the internet [7]. In our case, utilization of an online bariatric orientation requires that patients have access to the internet as well as be "eHealth literate", which requires not only literacy in the traditional sense, but also requires health, information, media, computer and scientific literacy [8]. US data from an international 2012 survey found that approximately 16 percent of the population was not digitally literate [9]. This survey found patients in the non-digitally literate group were on average older, had lower levels of education and were less likely to be active participants in the workforce. In regards to race and ethnicity, they found that the nondigitally literate group had a greater percentage of African Americans and Hispanics, which correlates with data from a 2016 Pew Research Center report indicating that Caucasians are more likely to use the internet when compared to blacks or Hispanics (3). These conclusions seem to pervade our group as well as we similarly found that the patients who chose the online seminar were on average, younger, less racially diverse with a higher percentage of 
Table 1 Demographics and clinical characteristics of patients who attended a clinical appointment, stratified by orientation type

\begin{tabular}{|c|c|c|c|}
\hline & Online $(n=187)$ & In person $(n=532)$ & $p$ value \\
\hline Age (Median, IQR) & $36.2(30.1-47.7)$ & $44.1(34.1-53.2)$ & $\mathrm{P}<0.001$ \\
\hline BMI (Median, IQR) & $45.4(40.8-51.2)$ & $45.3(41.6-50.2)$ & $P=0.78$ \\
\hline \multicolumn{4}{|l|}{ Gender $n(\%)^{*}$} \\
\hline Female, $n(\%)$ & $163(87.2)$ & $422(79.3)$ & \multirow[t]{2}{*}{$P=0.02$} \\
\hline Male, $n(\%)$ & $24(12.8)$ & $110(20.7)$ & \\
\hline \multicolumn{4}{|l|}{ Ethnicity $n(\%)$} \\
\hline Caucasian & $143(82.2)$ & $275(57.9)$ & \multirow[t]{7}{*}{$\mathrm{P}<0.001$} \\
\hline African American & $9(5.2)$ & $56(11.8)$ & \\
\hline Hispanic & $18(10.3)$ & $129(27.2)$ & \\
\hline Asian/Pacific Islander & $1(0.6)$ & $4(0.8)$ & \\
\hline Native American/Alaskan & $1(0.6)$ & $9(1.9)$ & \\
\hline Mixed & $2(1.1)$ & $2(0.4)$ & \\
\hline Unknown & 13 & 57 & \\
\hline \multicolumn{4}{|l|}{ Languages $n(\%)$} \\
\hline English & $180(96.8)$ & $434(83.5)$ & \multirow[t]{5}{*}{$<0.001$} \\
\hline Spanish & $4(2.2)$ & $64(12.3)$ & \\
\hline Bilingual & $1(0.5)$ & $18(3.5)$ & \\
\hline Other & $1(0.5)$ & $4(0.8)$ & \\
\hline Unknown & 1 & 12 & \\
\hline \multicolumn{4}{|l|}{ Marital status $n(\%)$} \\
\hline Married & $101(54.0)$ & $228(42.9)$ & \multirow[t]{4}{*}{$\mathrm{P}=0.04$} \\
\hline Divorced & $17(9.0)$ & $56(10.5)$ & \\
\hline Single & $66(35.3)$ & $235(44.2)$ & \\
\hline Unknown & 3 & 13 & \\
\hline \multicolumn{4}{|l|}{ Employment status $n(\%)$} \\
\hline Yes & $148(82.7)$ & $337(71.1)$ & \multirow[t]{2}{*}{$P=0.003$} \\
\hline Unknown & 8 & 58 & \\
\hline Previous abdominal surgery $n(\%)$ & $112(59.9)$ & $315(59.2)$ & $P=0.93$ \\
\hline $\mathrm{DM} n(\%)$ & $34(18.8)$ & $156(29.3)$ & $P=0.003$ \\
\hline $\mathrm{HTN} n(\%)$ & $67(35.8)$ & $290(54.5)$ & $\mathrm{P}<0.001$ \\
\hline Sleep apnea $n(\%)$ & $93(49.7)$ & $328(61.7)$ & $P=0.006$ \\
\hline Anxiety/Depression $n(\%)$ & $64(34.2)$ & $171(32.1)$ & 0.65 \\
\hline
\end{tabular}

$I Q R$-interquartile range, Values are presented as percentages
Caucasians over African American or Hispanic, and more likely to be employed. We also found that the patients who chose the online seminar were more likely to be married and had fewer comorbidities.

This information is particularly relevant at this time point as we are forced to rely exclusively on the use of web-based resources from everything from primary care visits to informational sessions. In the months leading up to the COVID pandemic, our online informational session had continued to increase in popularity, which we hoped would help increase overall access to our services. At this point we have had to put all in-person orientation sessions on hold and we are relying exclusively on our online orientation. Our pre-COVID data suggests that we may risk losing the patient population with traditionally lower rates of eHealth literacy. This means losing the patients who are older, are non-Caucasian and who have more comorbidities and less education, further propagating existing healthcare disparities.

Our study was limited by total number of patients and included only a single institution which limits the overall generalizability. It is also important to note that at the time of this study, the seminar we offered was only in English, which could have altered the demographics of the people who chose the OS over the IPS. We did perform a separate analysis with the English speaking patients only and essentially found the same differences in demographics with slightly altered p-values.

Despite these limitations, our study suggests that there are socioeconomic and clinical differences between the groups who chose the online seminar versus the in-person seminar. Ultimately our goal is to resume in-person orientation 
sessions after local and national social distancing regulations relax. In the interim, it is essential to target the disparities which we have identified in order to continue to improve and broaden access to bariatric surgery across our diverse patient population.

Supplementary Information The online version contains supplementary material available athttps://doi.org/10.1007/s00464-021-08453-7.

\section{Declarations}

Disclosures Drs Mia Shapiro, David Leenen, Beth Ryder, Todd Stafford, G. Dean Roye and Siva Vithiananthan have no conflicts of interest or financial ties to disclose.

\section{References}

1. Hales, Craig M, Carroll, Margaret D, Fryar, Cheryl D, Ogden, Cynthia L (2020) Prevalence of obesity and severe obesity among adults: United States, 2017-2018. NCHS Data Brief, no 360. Hyattsville, MD: National Center for Health Statistics

2. American Society for Metabolic and Bariatric Surgery (2019) Estimate of Bariatric Surgery Numbers, 2011-2018. http://asmbs. org/resources/estimate-of-bariatric-surgery-numbers

3. Brown, Anna, Lopez, G, Lopez MH. (2016). Digital Divide Narrows for Latinos as More Spanish Speakers and Immigrants go
Online. Pew Research Center's Hispanic Trends Project, Pew Research Center, www.pewresearch.org/hispanic/2016/07/20/ digital-divide-narrows-for-latinos-as-more-spanish-speakers-andimmigrants-go-online/.

4. Miletics M, Claros L, Stoltzfus J, Davis T, Chaar ME (2018) Progression to surgery: online versus live seminar. Surg Obes Relat Dis 14:382-385. https://doi.org/10.1016/j.soard.2017.11.014

5. Monfared S, Martin A, Gupta K, Stefanidis D, Selzer D, Choi J, Butler A, Banerjee A (2019) Web-Based Educational Seminars Compare Favorably with In-House Seminars for Bariatric Surgery Patients. Obes Surg 29:878-881. https://doi.org/10.1007/ s11695-018-3590-4

6. Eaton L, Walsh C, Magnuson T, Schweitzer M, Lidor A, Nguyen $\mathrm{H}$, Steele K (2012) On-line bariatric surgery information session as effective as in-person information session. Surg Obes Relat Dis. https://doi.org/10.1016/j.soard.2011.10.015

7. Lewis, Jamie (2017) "Internet Access and Racial/Ethnic Disparities.” The United States Census Bureau, www.census.gov/library/ working-papers/2017/demo/SEHSD-WP2017-31.html.

8. Norman CD, Skinner HA (2006) eHealth Literacy: Essential Skills for Consumer Health in a Networked World. J Med Internet Res 8:e9. https://doi.org/10.2196/jmir.8.2.e9

9. OECD Skills Outlook (2013) First results from the survey of adults skills. OECD Publishing, Paris

Publisher's Note Springer Nature remains neutral with regard to jurisdictional claims in published maps and institutional affiliations. 